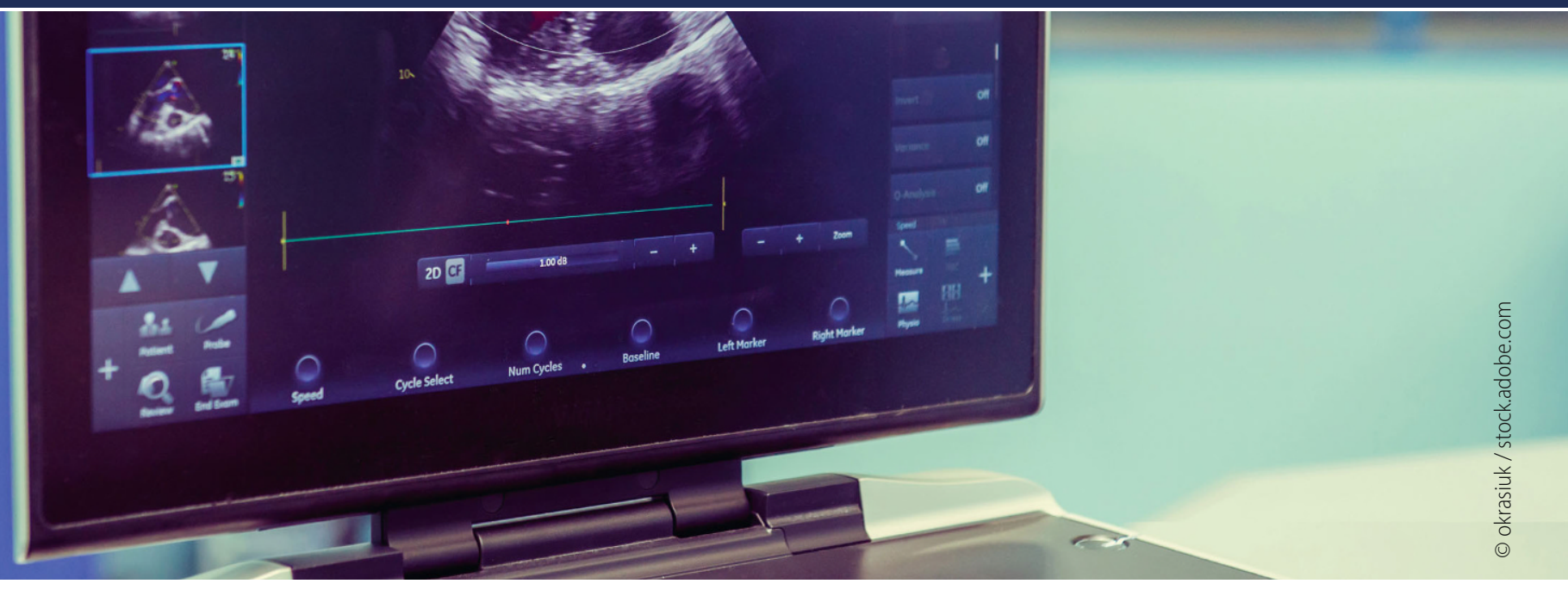

Gynäkologe 2022 ·55:4-6

https://doi.org/10.1007/s00129-021-04896-0

Angenommen: 2. Dezember 2021

Online publiziert: 17. Dezember 2021

(c) The Author(s), under exclusive licence to Springer Medizin Verlag GmbH, ein Teil von Springer Nature 2021

\section{Update fetale Echokardiographie}

\author{
Jan Weichert ${ }^{1}$ Klaus Diedrich ${ }^{2}$. Ulrich Gembruch ${ }^{3}$ \\ 'Bereich Pränatalmedizin und Spezielle Geburtshilfe, Klinik für Frauenheilkunde und Geburtshilfe, \\ Universitätsklinikum Schleswig-Holstein, Campus Lübeck, Lübeck, Deutschland \\ ${ }^{2}$ Klinik für Frauenheilkunde und Geburtshilfe, Universitätsklinikum Schleswig-Holstein, Campus Lübeck, \\ Lübeck, Deutschland \\ ${ }^{3}$ Abteilung für Geburtshilfe und Pränatale Medizin, Universitätsklinikum Bonn, Bonn, Deutschland
}

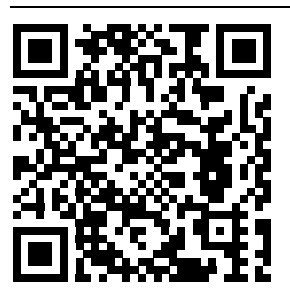

QR-Code scannen \&Beitrag online lesen
Die technischen Entwicklungen dieser zentralen und als Subdisziplin der pränatalen sonographischen Diagnostik weltweit etablierten Methodik sind seit den ersten Anwendungen zur fetalen Echokardiographie im A-Mode von Wang et al. im Jahr 1964 rasant vorangeschritten. In den frühen 1980er-Jahren konnten durch gezielte fetale 2-D-Echokardiographie erstmals verlässliche Aussagen zur Beurteilung fetaler Struktur- und Rhythmusanomalien getroffen werden. Etwa zur gleichen Zeit war es möglich geworden, mittels fetaler Doppler-Echokardiographie eine valide Beurteilung der Hämodynamik des fetalen HerzKreislauf-Systems und der Pathophysiologie fetaler kardiovaskulärer Erkrankungen vorzunehmen. Seit 2003 sind fetale kardiale Strukturen auch 3- bzw. 4-dimensional zu erfassen, was dem Untersucher einen direkten räumlichen Eindruck der kardiovaskulären Relationen im Herzen vermittelt. Zudem ist es mittlerweile möglich, bei bestimmten kardialen Läsionen therapeutische Interventionen in utero erfolgreich vorzunehmen. Mit der verbesserten Rechenleistungen moderner CPU (",central processing units") und der Entwicklung spezieller KI(künstliche Intelligenz)-Algorithmen hat auch die automatisierte Auswertung von 2-D-und 3-D/4-D-Daten Fahrt aufgenommen.

Mit diesem Themenheft von Der Gynäkologe möchten wir einen Überblick zum State of the Art und zu den perspektivischen Entwicklungen auf dem Gebiet der fetalen Echokardiographie bzw. der fetalen Kardiologie geben.

\section{॥) Mehrheit fetaler Herzfehler tritt im Low-risk-Kollektiv auf}

Obwohl sich die Entdeckungsraten von Herzfehlern in den vergangenen 10 Jahren in nationalen bzw. regionalen Screeningprogrammen tendenziell gebessert zeigten, liegt die Sensitivität nach wie vor zwischen 22,5 und $52,8 \%$. Lokale Richtlinien, die soziodemografische Situation der Eltern, der Zugang zum Screening, die Expertise der Untersucher sowie Art und Ausprägung des jeweiligen Vitiums sind höchstwahrscheinlich für die großen Unterschiede bei der vorgeburtlichen Diagnose von Herzfehlern verantwortlich. 
Hinzu kommt als maßgeblicher Faktor der Umstand, dass die überwiegende Mehrheit fetaler Herzfehler im Lowrisk-Kollektiv auftritt, nur 10\% in Schwangerschaften mit bekannten Risikofaktoren, wie erhöhte Nackentransparenz, positive Familienanamnese oder extrakardialen fetalen Fehlbildungen. Die Tatsache, dass bei etwa $60 \%$ der Neugeborenen mit einem kongenitalen Herzfehler ein chirurgischer oder kathetergestützter Eingriff erforderlich wird (bei der Hälfte der betroffenen Kinder innerhalb des ersten Lebensjahres), zeigt den Stellenwert einer detaillierten vorgeburtlichen kardialen Diagnostik.

Als Ursachen für die Nichterkennung eines kongenitalen Herzfehlers werden einer niederländischen Studie zufolge, u.a. eingeschränkte (visuomotorische) Anpassungsfähigkeiten des Untersuchers bei der sonographischen Erfassung der jeweiligen Herzebenen und eine gewisse Unkenntnis der jeweiligen Herzanomalien per se verantwortlich gemacht. Auch scheint eher ein logischer Ansatz zum Verständnis der anatomischen und pathophysiologischen Zusammenhänge von kardialen Vitien maßgeblich zu sein als ein rein theoretisches Wissen der einzelnen Läsionen. Unabdingbare Voraussetzung für das Vorangestellte bleibt eine standardisierte Darstellung der kardiologischen Referenzebenen, um entscheidende Informationen über die anatomische (räumliche) Anordnungen und deren Folgen für die funktionelle Integrität des Herzens erkennen zu können. Im Gegensatz zu den umliegenden Ländern, in denen im Zweittrimesterscreening auch die Darstellung der ventrikulären Ausflusstrakte und großen Arterien obligat ist, beschränkt sich das Screening in Deutschland noch immer auf die Darstellbarkeit des Vierkammerblicks und schließt somit die Diagnose vieler relevanter Vitien aus, wie Fallot-Tetralogie, Pulmonalatresie mit Ventrikelseptumdefekt, "double outlet right ventricle", Truncus arteriosus communis und Transposition der großen Arterien.

Bei der detaillierten Charakterisierung kardialer Vitien, insbesondere hinsichtlich perspektivischer Aussagen zum prä- und postnatalen Outcome, ist es längst nicht mehr ausreichend, eine alleinige Beschreibung der pathologisch veränderten Sonoanatomie des Herzens vorzunehmen. Untersuchungen zur Funktionsanalyse des fetalen Herzens sind hier in der Lage, zusätzliche, quantifizierbare Aussagen zur (eingeschränkten) Hämodynamik und Pumpleistung zu liefern. Es gibt dabei verschiedene Ansätze, die fetale Herzfunktion mit M-Mode, Dopplerflussanalyse und Gewebedoppler zu bewerten. Die Arbeitsgruppe um Christian Enzensberger und Oliver Graupner gibt in ihrem Artikel zur Funktionsdiagnostik einen dezidierten Up-to-date-Überblick über die Methoden der Beurteilung der systolischen und diastolischen Herzleistung. Dabei liegt der Fokus des Reviews auf der Analyse der regionalen und globalen Deformation des Herzens auf Basis der konventionellen 2-D-Echokardiografie mittels Speckle-Tracking.
Im Gegensatz zur konventionellen fetalen 2-D-Echokardiographie ist die kardiale Volumensonographie unter Verwendung der STIC (,spatial and temporal image correlation")-Technologie weniger abhängig vom Akquisitionswinkel durch Erfassung jeweils vollständiger Informationen der absoluten kardialen Dimensionen und somit auch weniger beeinflusst von der Lage des Feten in utero, da eine intuitive Erkennung und (Offline-)Rekonstruktion anatomischer Ebenen, die bei der 2-DEchtzeituntersuchung nicht sofort verfügbar sind, ermöglicht wird. Durch neuartige Sondentechnologien und Softwareentwicklungen konnte die kardiale Volumetrie v.a. hinsichtlich der STIC-Akquisitionszeit und Artefaktreduktion maßgeblich verbessert werden, dennoch bleibt die gezielte manuelle Navigation in einem Volumendatensatz anspruchsvoll. Alexander Weichert und Sven-Christian Weber gehen in ihrem Artikel auf diese und andere Fortschritte auf dem Gebiet der 3-D/4-DSonographie ein. Insbesondere zeigen sie, dass mittels spezieller, auf der Grundlage der STIC-Technologie entwickelter Algorithmen/Techniken, die den Benutzer bei der systematischen, workflowbasierten Analyse von Volumendatensätzen unterstützen, die segmentale Analyse der normalen und auffälligen kardialen Anatomie effizient und reproduzierbar möglich ist.

Anwendungen der KI haben mittlerweile auch in die pränatale sonographische Diagnostik Einzug gehalten. Während die automatisierte Biometrie (software-

Hier steht eine Anzeige. 
unterstützte Erkennung der für die Gewichtsschätzung notwendigen Parameter) herstellerübergreifend auf aktuellen Ultraschallsystemen generell verfügbar ist, ist die Zahl der klinisch nutzbaren KI-Algorithmen in der fetalen Echokardiographie eher noch überschaubar, wenngleich in den letzten Jahren eine Reihe erfolgversprechender Studien zum maschinellen Lernen publiziert wurden. Ein Blick in die Erwachsenenkardiologie zeigt, dass spezielle KI-basierte Analyse-Tools seit einigen Jahren (weiter)entwickelt und angewandt werden, die bereits jetzt eine direkte ("on the fly") Generierung und Quantifizierung wichtiger kardialer Variablen (u.a. Kammervolumen, Ejektionsfraktion) durch automatische Klassifizierung von 2-D-Schnittbildern ermöglichen. Einen Überblick zu den Einsatzmöglichkeiten der $\mathrm{KI}$ in der fetalen konventionellen 2-Dund 3-D-/4-D-Echokardiographie geben Jan Weichert et al. In Anlehnung an das Statement von George Box - „essentially, all models are wrong, but some are helpful" - werden hier das immense Potenzial automatisierter Algorithmen, aber auch deren (momentane) Limitierungen diskutiert. Die Autoren betonen in ihrem Beitrag, dass die interdisziplinäre Kommunikation und ständige Einbeziehung von Ärztinnen und Ärzten als Hauptanwender von KI-Technologien in die Entwicklungsprozesse und Funktionsweisen automatisierter Algorithmen unerlässlich sind. Dazu gehören u.a. die Supervision der Annotation und Kuratierung digitaler Daten (um diese überhaupt einer automatisierten Auswertung zugänglich zu machen), das Eintreten für tatsächlich relevante klinische KI-Lösungen und die Initiierung und Überwachung klinischer Studien zu KI-Algorithmen an großen Patientenkohorten.

In ihrem Artikel zu intrauterinen Therapieoptionen bei kongenitalen kardialen Vitien beschreiben Brigitte Strizek und Christoph Berg Details zur Kandidatenselektion und zur technischen Durchführung sowie die jeweiligen Risiken und das postnatale Outcome der einzelnen pränatalen Interventionen. Die Indikationen für ein fetales interventionelles Vorgehen bei schweren Ausflusstraktobstruktionen oder einem hypoplastischen Linksherzsyndrom (mit intaktem inter- atrialen Septum) sollten sich dabei am primären Ziel orientieren, wie der Beseitigung der Ausflussbahnobstruktion und dem Erhalt der Ventrikelfunktion oder, im Falle eines restriktiven Foramen ovale, durch eine frühzeitige Entlastung des Vorhofes potenziell einer Normalisierung der Lungenarchitektur.

Ein profundes Update zur Pathophysiologie, echokardiographischen Diagnostik und Therapie fetaler Rhythmusstörungen gibt Ulrich Gembruch in seinem Übersichtsartikel. Die korrekte Erfassung und Analyse fetaler Arrhythmien mittels M-Mode und Dopplersonographie ist nach wie vor anspruchsvoll. Im Gegensatz zu den harmlosen supraventrikulären Extrasystolie sollten anhaltende Tachyarrhythmien transplazentar antiarrhythmisch behandelt werden, dabei beeinflusst der Typ der Tachyarrhythmie die Therapiewahl. Die pränatalen therapeutischen Optionen beim immunologisch induzierten kompletten kongenitalen AV-Block bleiben dagegen eingeschränkt bzw. sind umstritten (transplazentare Behandlung mit hochdosierten fluorierten Kortikosteroiden). Bei belasteter Anamnese - vorheriges Kind mit immunologisch induziertem kompletten AV-Block - scheint die orale Gabe des plazentagängigen Hydroxychloroquin (HCQ) die Häufigkeit eines erneuten Auftretens eines CCHB im Hochrisikokollektiv signifikant um rund $50 \%$ zu senken. Die perorale Applikation von 200-400 mg HCQ sollte dabei spätestens bis zur 10. SSW begonnen werden.

Zusammenfassend lässt sich sagen, dass sich die fetale Echokardiographie gerade in den letzten Jahren des 20. Jahrhunderts zur wichtigsten Modalität für die Diagnose, Prognose und Behandlung von Feten mit kardiovaskulären Erkrankungen entwickelt hat. Im multidisziplinären Setting können so die Beratung und Betreuung von Feten mit strukturellen, funktionellen oder rhythmischen Herzanomalien effektiv und individuell gewährleistet werden. Der zu Recht oder zu Unrecht Albert Einstein zugesprochenen Feststellung - „Die Definition von Wahnsinn ist, immer wieder das Gleiche zu tun und andere Ergebnisse zu erwarten" - folgend, sollte auf dem Weg hin zu einer weiter verbesserten pränatalen Detektionsrate kardialer Vitien und damit zu einem gezielten individualisierten perinatalen Management die Implementierung von Neuerungen in der sonographischen Bildgebung und -analyse konsequent verfolgt werden. Für Deutschland wäre zudem neben einer entsprechenden Schulung der Untersucher dringend eine Änderung der Screeningvorgaben erforderlich, indem das Zweittrimesterscreening durch die obligate Darstellung der ventrikulären Ausflusstrakte mit den Abgängen der großen Arterien erweitert würde.

\section{Korrespondenzadresse}

\section{Prof. Dr. Jan Weichert}

Bereich Pränatalmedizin und Spezielle Geburtshilfe, Klinik für Frauenheilkunde und Geburtshilfe, Universitätsklinikum SchleswigHolstein, Campus Lübeck

Ratzeburger Allee 160, 23538 Lübeck, Deutschland

jan.weichert@uksh.de

Interessenkonflikt. J. Weichert, K. Diedrich und $U$. Gembruch geben an, dass kein Interessenkonflikt besteht. 